\title{
Parkinson's disease psychosis
}

This article was published in the following Dove Press journal:

Journal of Parkinsonism and Restless Legs Syndrome

27 March 2014

Number of times this article has been viewed

\author{
Rebekah J Jakel ${ }^{1,2}$ \\ Mark Stacy ${ }^{3}$ \\ 'Department of Psychiatry, Duke \\ University, Durham, NC, USA; \\ 2Durham Veteran's Administration \\ Medical Center, Durham, NC, USA; \\ ${ }^{3}$ Department of Neurology, Duke \\ University, Durham, NC, USA
}

Correspondence: Mark Stacy

Duke University Medical Center, 932 Morreene RD, MS 3333,

Durham, NC 27705, USA

Email mark.stacy@duke.edu
Abstract: Parkinson's disease is a progressive neurodegenerative disease characterized by loss of nigral dopaminergic neurons associated with rigidity, resting tremor, bradykinesia, and postural instability. In addition to the above motor symptoms, nonmotor manifestations are increasingly recognized as part of Parkinson's disease pathology and contribute to overall symptom burden, morbidity, and mortality. Such nonmotor symptoms include autonomic dysfunction, impaired olfaction, gastrointestinal disturbances, and a variety of psychiatric symptoms including psychosis. Psychiatric symptoms may be inherent to the disease process itself, secondary to treatments aimed at restoring dopamine, or related to comorbid mental illness. Given that traditional medications used to treat psychosis are dopaminergic antagonists, pharmacologic treatment of these symptoms carries the risk of worsening the movement disorder, creating a challenge for providers. This review examines current literature regarding psychosis in the context of Parkinson's disease including risk factors for psychosis, prognosis, and management of these challenging symptoms.

Keywords: antipsychotics, delusions, hallucinations, non-motor symptoms, paranoia

\section{Introduction}

Parkinson's disease (PD) is a progressive neurodegenerative illness associated with loss of dopaminergic neurons in the substantia nigra. PD is characterized by rigidity, resting tremor, and bradykinesia; the classic triad of symptoms or cardinal features that define the movement disorder. Increasingly, the nonmotor features of PD (including autonomic dysfunction, impaired olfaction, and gastrointestinal disturbances, as well as psychiatric symptoms such as sleep disturbances, depression, impulse control disorders, dementia, and psychosis) are being recognized as important features associated with this condition (Table 1). Indeed, psychiatric manifestations of PD can have great impact on the lives of patients with PD, as well as on those of their caregivers. This review examines the current literature regarding psychotic symptoms in PD.

Psychotic symptoms that occur in the context of PD, or PD psychosis (PDP), is a recognized symptom cluster. PD patients can experience a variety of psychotic symptoms; however, these symptoms most commonly include visual hallucinations, delusions of jealousy, or milder false sense of presence and illusions. PDP symptoms can exacerbate the debility of PD and create complexities in the care of patients with PD. Research suggests that development of psychotic symptoms is associated with worsening quality of life for the patient and, frequently, a remarkable increase in caregiver burden. ${ }^{1}$ Furthermore, the presence of psychotic symptoms may be associated with 
Table I Symptoms of Parkinson's disease (PD)

\begin{tabular}{ll}
\hline Motor symptoms of PD & Non-motor symptoms \\
\hline Resting tremor & Constipation \\
Rigidity & Autonomic dysfunction \\
Bradykinesia & Impaired olfaction \\
Postural instability & Dementia \\
Festinating gait & Depression \\
Micrographia & Sleep disorders \\
Masked facies & Impulse control disorders \\
Retropulsion & Psychosis \\
Hypophonic speech & \\
\hline
\end{tabular}

worsening outcomes, including necessity of nursing home placement $^{1}$ and increased mortality. ${ }^{2}$

\section{Psychotic symptoms in PD}

Psychotic symptoms in PD may include hallucinations, illusions, paranoia, and a false sense of presence. The quality of psychotic symptoms in PDP differs from that of primary psychotic disorders such as schizophrenia. Most patients with PDP have retained insight and clear sensorium initially, which are lost over time. Psychotic symptoms typically arise later in the course of the illness, such that prominent hallucinations that occur early in the course of a movement disorder should alert the clinician to consider Lewy body dementia (LBD), Alzheimer's disease variants, delirium, or other psychiatric disease (such as major depression with psychosis) in the differential diagnosis.

\section{Illusions}

Illusions are misperceptions of actual objects. For example, a curtain may be perceived as a person. This differs from hallucinations in which no object is present to be misinterpreted. Illusions are not uncommon phenomena and are considered to be more benign than frank visual hallucinations. Clinical experience and limited data suggest that illusions may be more likely to emerge with declining cognition, impaired vision, poor lighting, and with patient fatigue. ${ }^{3}$ Patients may demonstrate retained insight.

\section{False sense of presence}

Many patients report the sensation of another person being in the room with them when they are alone. This has been coined a false sense of presence. Similar to illusions, this is considered to be a benign phenomenon; however, repeated correction from primary caregivers, in response to false sense of presence, may lead to paranoia and belief that a spouse is being unfaithful, a frequent delusion in PD. Indeed, preliminary work suggests that most patients with benign psychotic symptoms will progress to more severe psychotic symptoms, suggesting a role for monitoring of all forms of psychosis. $^{4,5}$

\section{Hallucinations}

Hallucinations, or sensory experiences in the absence of external stimuli, can also be present in PDP. The features of hallucinations found in the context of PD differ from those found as part of primary psychotic disorders such as schizophrenia and schizoaffective disorder, which are primarily auditory. Hallucinations in PD tend to be visual as well as auditory. Prominent visual hallucinations seen early in the course of illness, with clouded sensorium and minor Parkinsonism features, are strongly suggestive of LBD.

Like other forms of psychosis, insight is typically retained early in the emergence of hallucinations. As the disease progresses, insight is generally lost. Not all patients with hallucinations voluntarily report them or seek treatment. Early data examining factors that prompt patients with visual hallucinations to seek care suggests that individual perception, or even value, of the hallucinatory experience may influence the decision to report or seek treatment for these phenomena. ${ }^{6}$

\section{Delusions}

Delusions are another form of psychotic thought content in PD. Delusions are fixed, false beliefs. They can relate to nearly any situation that has valence in one's life, but are most often paranoid in nature. There is less literature relating to delusional symptoms in PD; however, case reports of Othello syndrome (pathologic jealous delusions of marital infidelity) have followed diagnosis of PD. ${ }^{7,8}$ In these cases, reduction in dopamine agonist treatment and/or the addition of an antipsychotic were effective in treating the psychosis. Similarly, there are isolated reports of patients with PD who develop delusional parasitosis: the false belief of being infested with a parasite. This also remitted with cessation of dopamine agonists. ${ }^{9}$ The average age of patients experiencing delusional symptoms is younger than that of those experiencing hallucinations, suggesting an etiology of delusions that may be phenomenologically different to that of hallucinations. ${ }^{10}$

\section{Prevalence}

The prevalence of psychotic symptoms in PD is difficult to determine, primarily because of nonuniform methods of defining and measuring symptoms of psychosis in PD. Many rating scales are used to screen PD patients for psychotic symptoms including the Unified Parkinson Disease Rating 
Scale Thought Disorder, the Brief Psychiatric Rating Scale, the Clinical Global Impression of Improvement, and the Parkinson's Psychosis Rating Scale. Rating scales vary in terms of the training required to administer, availability of validated cut-offs, and criteria for use. For this reason, literature estimates of the prevalence of PDP vary widely. Current figures are as high as $60 \% .{ }^{11-14}$ A 12 -year study of development of psychosis in PD revealed that, at the end of the study period, $60 \%$ of patients showed psychotic symptoms, with an incidence of 797 per 1,000 person-years. $^{2}$

It should be noted that, given the complexity of studying something that does not have universally accepted criteria, there have been attempts to create a universal definition of PDP. Prior to the publication of Diagnostic and Statistical Manual, Fifth Edition (DSM-5), an expert consensus group proposed criteria for PDP to distinguish it from the Diagnostic and Statistical Manual, Fourth Edition (DSM-IV) text revision classification of "psychosis due to a general medical condition." 15 This group defined PDP as the presence of psychotic symptoms for at least a month, due to PD (and not another cause), with or without retained insight, with or without dopaminergic medication use. Further discussion follows regarding diagnostic criteria for PDP.

\section{Risk factors for PDP}

Psychosis is not universally found in all patients with PD. Consequently, there have been attempts to further understand possible risk factors for PDP. Factors that likely impact the development of PDP include age of onset, presence and severity of cognitive impairment, presence of sleep disorders and other psychiatric symptoms, and medications (Table 2).

The PRIAMO study, a large multisite Italian trial, enrolled 495 patients with early stage PD (Hoehn and Yahr $\leq 2$, median duration 3.4 years). ${ }^{16}$ Patients were followed for 2 years as part of a longitudinal observational study. The baseline prevalence of psychotic symptoms was 3\%, which increased to $5.2 \%$ at 1 year, and $7.7 \%$ at the end of the observation

Table 2 Likely risk factors for development of psychosis in Parkinson's disease

Advancing age

Cognitive decline

Duration of illness

Severity of illness

Treatment with dopaminergic agonists

Presence of comorbid sleep disorders

Presence of other psychiatric symptoms (apathy, depression) period. Psychotic symptoms were associated with cognitive decline.

A neurogenetics research consortium studying susceptibility genes in PD enrolled 500 patients with PD. ${ }^{17}$ A multivariate cross-sectional analysis of data obtained from this sample demonstrated that, in terms of motor symptoms, psychotic symptoms were associated with freezing of gait (odds ratio [OR] 3.83; 95\% confidence interval [CI], 1.678.75; $P<0.002$ ) but not tremor, postural instability, specific polymorphisms of genes such as Alpha-synuclein, microtubule associated protein tau genes, or apolipoprotein.

\section{Age of onset}

Features associated with the onset of PD also likely impact prevalence of PDP. Some data suggest that older age of onset is more frequently associated with PDP when compared to younger-onset variants. In a large single-site series, consecutive patients diagnosed with PD in a neurology outpatient clinic were stratified into early-, middle-, and late-onset PD $(\mathrm{N}=230)$, and motor and nonmotor symptoms of PD were assessed. Hallucinations, but not delusions, were associated with older age of onset (OR 5.32; 95\% CI, 1.78-15.97). ${ }^{18}$ Another retrospective chart review of 1,453 Japanese patients in an outpatient neurology clinic similarly showed that psychotic symptoms were more likely to occur in patients with late-onset PD $(P<0.0001) .{ }^{19}$ In this study, an average of $28.6 \%$ of patients had psychotic symptoms an average of 9 years after onset of PD, with $53.9 \%$ of late-onset and $22.1 \%$ of early-onset patients demonstrating psychosis by year 12 $(P<0.001){ }^{19}$

\section{Cognitive impairment}

The presence of psychotic symptoms in PD also appears to vary with degree of cognitive impairment. A study of the prevalence of psychosis in PD in those without any cognitive impairment, as compared to those with mild cognitive impairment or dementia, demonstrated that psychotic symptoms positively correlated with severity of cognitive impairment; ${ }^{20}$ this was also found in the PRIAMO trial. ${ }^{16}$

In attempts to develop specific criteria for psychotic symptoms without confounding cognitive impairment, 250 patients with idiopathic PD and mini-mental status examination (MMSE) $>23$ from community-based clinics were evaluated for psychotic symptoms based on National Institute of Neurological Disorders and Stroke/National Institute of Mental Health criteria. ${ }^{21}$ Of the total sample, 26\% had current psychotic symptoms with less than half of those having minor symptoms such as illusions and false sense of 
presence. Similarly, another study examined the frequency of psychotic symptoms in nondemented PD patients $(\mathrm{N}=191)$ as referred from outpatient clinics. ${ }^{22}$ Psychotic symptoms were evident in $21.5 \%$, and were most commonly visual hallucinations and illusions/misidentification of people. This suggests that, though psychotic symptoms are more common in the context of dementia, patients without dementia can demonstrate PDP, and these findings support screening for PDP in all PD patients.

\section{Disease duration and severity}

Another case-control study looked at patients with PD who survive greater than 20 years with the illness (as compared to matched patients of 10-15 year duration). ${ }^{23}$ They found that long-duration of disease was correlated with younger age at onset $(P<0.0001)$ and had a higher incidence of psychosis $(P<0.038)$ and left handedness/left-sided symptom onset. Rates of dementia and tremor were similar between groups. A multivariate cross-sectional analysis of characteristics of 500 patients with PD showed that psychotic symptoms were associated with increasing age (OR 4.86; 95\% CI, 1.62-14.30) as well as duration of disease (OR 3.81, 95\% CI, 1.23-11.76). ${ }^{17}$

\section{Psychiatric symptoms}

The PRIAMO study of early stage PD patients also showed that psychotic symptoms were correlated with presence of sleep disturbances, treatment with dopamine agonists, disease severity, and depressive and anxiety symptoms. ${ }^{16}$ In a nondemented population, depression and rapid eye movement behavior disorder were also found to correlate with psychotic symptoms. Patients with comorbid depression or sleep-wakefulness demonstrated a five times greater odds of psychotic symptoms. ${ }^{22}$

\section{Dopamine agonist administration}

Patient- and medication-related risk factors for psychosis in PD were examined in a retrospective cohort of 331 PD patients who were followed for 2 years. ${ }^{24}$ The inclusion criteria included past diagnosis of PD, current dopaminergic therapy, and a past history of psychosis. Exclusion criteria included: treatment with an antipsychotic medication in the month prior to the study, a history of schizophrenia, or patients with known or probable LBD. In those patients who developed psychosis (defined as seeking care for symptoms resulting in a prescription for an antipsychotic therapy), the medications used during the period 14 days prior to onset of psychosis were compared to those administered 1 and
3 months prior to onset, which was deemed the control period. The incidence of psychosis was found to be 116 per 1,000 person-years (95\% CI, 85-148), with increased risk in patients with more advanced $\mathrm{PD}$, longer duration of $\mathrm{PD}$, and lower MMSE scores ( $\leq 24)$. In terms of medications, anticholinergic treatment significantly elevated risk of PDP (hazard ratio [HR] 19.7; 95\% CI, 2.39-162) and donepezil use reduced the risk (HR 0.48; 95\% CI, 0.25-0.85). The dose of dopaminergic drugs positively correlated with risk of psychosis in older patients (HR 1.65; 95\% CI, 1.02-2.66). This risk was more pronounced in older patients ( $>70$ years) as medication-related risk was not found in young patients.

\section{Etiology}

The etiology of PDP is complicated and may be multifactorial. Possible explanations for PDP include: 1) PDP could be inherently related to the pathogenesis of PD; 2) PDP could be iatrogenic due to treatment with anticholinergic medications, dopamine prodrugs, and dopamine agonists; or 3) psychosis in patients with $\mathrm{PD}$ could be related to a comorbid psychiatric disorder that may or may not be interacting with PD. Much of the literature focuses on the relationship between treatment with dopaminergic agents and the emergence of psychosis. Indeed, treatment with dopaminergic agonists is strongly associated with emergence of PDP. ${ }^{25}$ However, PDP may be a feature of PD independent of dopaminergic treatment, or PD may be a risk factor that confers sensitivity to psychosis exacerbated by treatment medications. These factors are not mutually exclusive and can be difficult to study.

\section{Psychosis as a feature of the PD}

The incidence of psychotic symptoms in patients with untreated PD as part of the natural course of the disease is considered to be low; however, in the current treatment paradigm, almost all patients receive dopaminergic therapy, and these agents are highly associated with psychosis. In addition, examination of historical data prior to the levodopa era is confounded by use of anticholinergic and ergot compounds and lack of prospective data. Nonetheless, Fenelon et $\mathrm{al}^{26}$ have suggested that that there may be a natural increase in hallucinations in late-stage PD.

Given the multitude of treatments available for PD, it is not ethical to study the natural course of untreated PD, limiting our understanding of the pathogenesis of PDP in the absence of dopaminergic or other agents. Experimental imaging of patients with suspected PD prior to treatment may offer some clues. Ravina et al used dopamine transporter (DAT) imaging with $\left.{ }^{123} \mathrm{I}\right][\beta]$-CIT and single-photon 
emission computerized tomography (SPECT) to evaluate a group of patients with suspected PD, prior to initiation of any dopaminergic medication at baseline, and subsequently at 22 months. ${ }^{27}$ Lower rates of striatal DAT binding at baseline predicted both motor and nonmotor impairment, including development of psychosis. DAT binding was distributed into quartiles, and those in the lowest quartile, as compared to the top, had an OR of 12.9 (95\% CI, 2.6-62.4) for psychosis. ${ }^{27}$ This suggests that factors exist prior to dopaminergic therapy that may influence development of nonmotor symptoms. However, in preclinical stages, use of imaging may aid in predicting progression of illness in the future. It may also allow for development of novel treatments.

Loss of dopaminergic pathways is a key feature of PD, and may play a role in the development of psychosis. However, other neurotransmitter pathways, including cholinergic and serotonergic pathways, may also be involved in the generation of psychotic symptoms in PD. ${ }^{28}$ Serotonergic neurons, largely originating in the dorsal raphe nuclei, are also lost in PD and can demonstrate Lewy body formation similar to the histopathologic feature found in dopaminergic neurons. There is also evidence that serotonin neurons are lost prior to degeneration of the midbrain dopaminergic pathways. ${ }^{29}$ Serotonin $5-\mathrm{HT}_{2 \mathrm{~A}}$ receptors have been implicated in the pathogenesis of hallucinations and could play a role in PDP. Studies have attempted to correlate pathology of the serotonergic system with clinical manifestations of PD in a patient population, ${ }^{28}$ but small sample sizes have precluded formal conclusions.

\section{Treatment-emergent PDP}

Psychotic symptoms in PD have typically been thought to be iatrogenic; medications used to replace nigrostriatal dopamine also increase dopamine in areas of the brain that are responsible for the generation of psychotic symptoms. There is increasing evidence that treatment with dopaminergic agents predates onset of psychotic symptoms. A chart review of 10,347 patients of at least 66 years of age who were started on dopaminergic therapy for PD examined the emergence of psychotic symptoms as defined by treatment with antipsychotic. This study revealed that the estimated cumulative probability of requiring an antipsychotic at 7 years was $35 \%$. Nearly $5 \%$ of individuals started on dopaminergic therapy were prescribed an antipsychotic within a year of initiation of dopaminergic treatment (4.8\%; 5.2/100 person-years), ${ }^{30}$ thus supporting that dopaminergic treatment plays a role in the development of hallucinations.
Furthermore, a cross-sectional retrospective study of PD patients with psychosis versus age-matched controls without psychosis showed that the odds of psychosis were highest in the population treated with the dopamine agonist pergolide (when controlling for other factors thought to be associated with PDP including age and dementia [OR 2.01, 95\% CI, 1.22-5.45]). ${ }^{25}$ Emergence of psychotic symptoms was lowest with levodopa (OR 0.11 ; 95\% CI 0.06-0.19).

Although pharmacologic treatments have been considered the main offender in the genesis of PDP, the advent of nonpharmacologic biologic treatments for PD has also revealed a possible association in the development of PDP. A case report describes new-onset florid psychosis after placement of bilateral subthalamic nucleus deep brain stimulators (DBS) in two patients with young-onset PD, without prior psychiatric history. ${ }^{31}$ Both patients had been unable to tolerate higher levels of oral dopaminergic medications due to emergence of hypersexuality and other compulsive behaviors and, thus, were referred for DBS. Neither patient had evidence of postoperative delirium; however, both became psychotic prior to activation of the stimulator. They both required parenteral antipsychotics (olanzapine and haloperidol) to manage the psychosis, without worsening of movement symptoms. ${ }^{31}$ This suggests that the lesion effect of DBS placement may have therapeutic implications. Furthermore, the initial inability to tolerate dopaminergic medications may suggest an underlying vulnerability to psychosis given the emergence of side effects across treatment modalities.

\section{Comorbid preexisting psychiatric disease and PD}

Although rare, it is possible for patients with psychiatric disorders to develop PD as the conditions are not mutually exclusive. A recent cross-sectional study examined frequency of comorbidities diagnosed in patients with schizophrenia. ${ }^{32}$ It found that, compared to control individuals, people with schizophrenia were more likely to have PD as a comorbidity with an OR of 3.07 (95\% CI, 2.42-3.88). Given the limitations of the study design, the nature of this relationship remains difficult to describe. Clearly, antipsychotic treatment for schizophrenia can cause parkinsonism (as compared to PD), and further delineation of either the schizophrenia or PD diagnosis cannot be determined from the study details. There is little data on the incidence of PD as a comorbidity of other psychiatric disease with psychosis such as bipolar affective disorder or major depression with psychotic features. 


\section{Pathophysiology}

There is limited research into understanding how psychotic symptoms, namely hallucinations, occur in PD. Researchers have attempted to use neuropsychological testing and imaging to better elucidate psychotic features in PD. Neuropsychological correlates of hallucinations were studied in 57 PD patients, with and without visual hallucinations, who were matched for age, education, and disease duration. ${ }^{33}$ Cognition was measured using 2 instruments: the PD-Cognitive Rating Scale and the Mattis Dementia Rating scales. In the specific cognitive domains examined, subjects with minor visual hallucinations did not differ significantly from the absence of minor symptoms. Of those patients with major visual hallucinations, the presence of retained insight was associated with predominantly frontal-striatal impairment. Those without insight demonstrated more extensive neuropsychiatric deficits, including in tasks involving more posterior cortical areas, not seen in those patients who had retained insight. Perhaps, because of the sample size, this was not correlated with imaging.

Neuroimaging with proton magnetic resonance spectroscopy was used to examine the degree of neuronal integrity in the anterior cingulate cortex,$^{34}$ a brain region that has been implicated in executive function. PD patients without dementia $(\mathrm{N}=20)$ demonstrated decreased neuronal integrity in the anterior cingulate cortex as compared to healthy controls $(\mathrm{N}=20)$. This was not seen in the posterior cingulate cortex (a control region). This also correlated with worsening cognitive functioning including set-shifting and presence of psychosis, which is consistent with data showing a relationship between cognition and hallucinations as described above.

\section{Management of psychotic symptoms in PD \\ Differential diagnosis and assessment}

As previously mentioned, not all patients with psychosis seek treatment on their own accord. The concern for psychotic symptoms in PD frequently comes from family and patient caregivers; therefore, accurate assessment and diagnosis is strongly reliant upon collateral information from those who can give appropriate historical details, in addition to direct evaluation of the patient. Initial management of psychotic symptoms in PD includes complete medical evaluation to rule out other causes of hallucinations (Figure 1). Delirium is a form of altered mental status caused by underlying medical illness such as infection, hypoxia, or electrolyte imbalances. It is characterized by fluctuation in sensorium, with impaired attention and concentration, and can include hallucinations (primarily visual, but also tactile and auditory), delusions, and paranoia. Risk factors for delirium, including advancing age and preexisting brain disease, are inherent to $\mathrm{PD}$, and delirium is not an uncommon phenomenon when patients with PD have worsening comorbid health concerns. The time course of delirium - which is usually more acute, or subacute, and coincides with medical illness - helps distinguish it from psychosis second to PD. However, because patients may also have underlying dementia, the two may be difficult to distinguish, and delirium may go unrecognized. Delirium is treated primarily by addressing the underlying medical cause(s); improvement in mental status may lag behind clinical resolution of the underlying cause.

The differential for psychotic symptoms in context of PD also includes other forms of dementia such as LBD, Alzheimer's disease, and vascular dementia. In LBD, neuropsychiatric symptoms typically precede mild motor dysfunction and are characterized by dementia, visual hallucinations, and stupor. Patients with LBD also tend to exhibit more symptoms of agitation and often require the assistance of more than one caregiver, particularly for clinic appointments. Other forms of dementia may also have features of parkinsonism and hallucinations. Psychotic symptoms in a patient with parkinsonism can also be related to a primary psychiatric disorder such as schizophrenia, schizoaffective disorder, or psychotic depression that is comorbid with PD. Alternatively, motor symptoms in a patient with a primary psychotic disorder may be second to tardive parkinsonism, which is an effect of prolonged exposure to high potency dopamine receptor blocking medications. Features such as auditory hallucinations (with command nature) or presence of mania should alert the clinician to consider a psychiatric cause.

\section{Treatment}

Although psychotic symptoms may be phenomenologically related to PD pathogenesis, the vast majority of patients with PDP are treated with some form of dopaminergic agent to relieve motor symptoms. This necessary therapeutic approach puts patients at risk of iatrogenic cause, or worsening, of psychotic symptoms in PD. Consequently, the first-line treatment in PDP is to reduce or eliminate polypharmacy; simplification of the array of dopaminergic or other adjunctive medications may control the psychotic symptoms. Reduction of anticholinergic medications for general medical conditions, particularly for treating bladder, sleep, or cold or allergy 


\section{Psychotic symptoms in patient with} PD diagnosis

Full medical evaluation (history and physical) to rule out delirium or other causes of psychosis

- Routine labs

- CBC, chem 7, LFTs, $\mathrm{NH}_{3}$, UA, TSH, RPR, ECG, CXR if clinical suspicion

- Caregiver collateral

Treat any positive results as appropriate and continue to monitor for psychotic symptoms

[Assuming no delirium or other cause found, or if psychosis continues after resolution of delirium]

Minimization of medications for general medical conditions, such as bladder conditions, sleep, or allergies, or of cold medications (anticholinergic, antihistamines)

[If no improvement of symptoms]

Reduction or elimination of adjunct antiparkinsonian medications (MAO inhibitors, amantadine, trihexyphenidyl)

[lf no improvement of symptoms]

Reduction or discontinuation of dopamine agonists (may need to replace with levodopa), or reduction of levodopa if not on dopamine agonist

[If no improvement of symptoms or worsening of motor symptoms]

Initiation of low dose of quetiapine $12.5 \mathrm{mg}$ po qhs to titrate with counseling of risks, black box warnings, and appropriate monitoring

[If no improvement of symptoms or worsening of motor symptoms]

Initiation of clozapine at low dose of $6.25 \mathrm{mg}$ qhs to titrate slowly, with counseling of risks, black box warnings, and mandated monitoring

Figure I Treatment algorithm for treating PDP.

Abbreviations: $C B C$, complete blood count; chem 7, basic metabolic test; CXR, chest X-ray; LFTs, liver function tests; ECG, electrocardiography; MAO, monoamine oxidase; $\mathrm{NH}_{3}$, ammonia; PD, Parkinson's disease; PDP, Parkinson's disease psychosis; po, per oral; qhs, at night; RPR, rapid plasma reagin (syphilis); TSH, thyroid stimulating hormone; UA, urinalysis.

symptoms, is necessary. A second step is to minimize (or discontinue) adjuvant PD medications such as anticholinergic medications, amantadine, and monoamine oxidase $\mathrm{B}$ inhibitors to the lowest effective doses. If this fails to reduce psychotic symptoms, reduction of dopaminergic agents may be warranted. Given the high potential for worsening mobility, any reductions in dopaminergic therapy should be completed with close follow-up evaluations. As dopamine agonists may be more likely to cause psychotic symptoms, it may be useful to start with tapering and/or discontinuation of these medications first, with reductions in levodopa dosing as a second line approach.

If alterations in dopaminergic agonists or levodopa dosing are not successful in reducing psychotic symptoms or 
aggravate motor function, introduction of medication to treat psychotic symptoms should be considered. Optimally, treatment with an antipsychotic would reduce or eliminate psychotic symptoms without coincident worsening of movement and symptoms. However, the potent dopamine blockage provided by traditional antipsychotics in the nigrostriatal pathway can cause extrapyramidal side effects and put patients at risk for marked worsening of mobility. At present, clozapine and quetiapine are the two agents considered to be least likely to worsen motor function while treating psychotic symptoms. It is important to note that once psychotic symptoms emerge they are likely to continue. ${ }^{4}$ Although it would be ideal to use antipsychotic medications for a short time, slow weaning from antipsychotics frequently leads to recurrence of symptoms as quickly as 8 weeks. ${ }^{35}$

There are many antipsychotic medications on the market, and they differ in potency in relation to dopaminergic receptors and other receptor types, such as serotonin. In general, first generation, or traditional antipsychotics, are contraindicated given their relative potency for dopaminergic receptors, and should be avoided. To further guide clinical decision making, the Movement Disorder Society published an evidence-based review of treatments for the nonmotor symptoms of PD in $2011 .{ }^{36}$ Recommendations were made based on available evidence, with preference for appropriately powered double-blind placebo-controlled trials. Of those medications on the market that have been studied for PDP, it was concluded that clozapine was efficacious, with "acceptable risk with specialized monitoring." ${ }^{36}$ Quetiapine was concluded to have "insufficient evidence" for efficacy with "acceptable risk without specialized monitoring." 36 Olanzapine was considered unlikely to be efficacious with "unacceptable risk" second to worsening movement symptoms. ${ }^{36}$ Given the degree of dopaminergic blockade, other antipsychotics are likely to cause worsened motor symptoms.

Clozapine is the gold-standard treatment for psychosis due to schizophrenia, and likely for treating PDP. Despite its efficacy, it is associated with the serious life-threatening adverse event of agranulocytosis in $1 \%$ of individuals who take the medication, which severely limits its use in general practice. Clozapine administration is highly regulated and requires a patient registry as well as provider registration with the Food and Drug Administration for authorization to write prescriptions. Patients are mandated to weekly to biweekly monitoring of white blood cell counts to monitor for agranulocytosis. Other antipsychotics have risks of rare blood dyscrasias, but none to the degree of risk incurred by clozapine. In addition to agranulocytosis, clozapine has risks of toxic megacolon and cardiomyopathy. The doses typically used in PDP are on the order of $50 \mathrm{mg}$, which is much less than doses used for patients with primary psychosis.

Because of the risks of clozapine, most clinicians will prescribe quetiapine as an initial choice. Although there is less risk associated with quetiapine administration, data from several studies, including a small randomized placebocontrolled trial, suggest that it is likely not as efficacious as clozapine in PDP. ${ }^{37-39}$

Use of antipsychotic medications in the elderly is fraught with risks in addition to potential worsening of motor symptoms. Atypical antipsychotics carry black box warnings for increased all-cause mortality for use in the elderly with dementia. Other side effects include postural hypotension, increased falls risk, dizziness, metabolic side effects, decreased seizure threshold, prolonged corrected QT interval (QTc), and drowsiness. The risks of antipsychotic use in PD were examined in a community-based sample of Norwegian PD patients followed for $>12$ years. ${ }^{2}$ This study demonstrated that mortality was predicted by presence of psychosis. However, treatment with antipsychotic medication did not appear to have significant impact on survival in this cohort (HR 1.05; 95\% CI 0.70-1.58, $P=0.807$ ).

The risks of antipsychotics have prompted researchers to examine the utility of other non-neuroleptic medications to target psychotic symptoms in PD. There are few randomized controlled trials, but several case reports of off-label use of a variety of psychotropics to treat psychotic symptoms in PD. One case report detailed use of mirtazapine, a newgeneration antidepressant, to successfully treat visual hallucinations in a patient with PD without concurrent worsening of motor function. ${ }^{40}$ However, in general, there is insufficient data to recommend treatment with antidepressants for PDP.

Electroconvulsive therapy (ECT) is a gold-standard biologic therapy for severe depression, and can also be useful in other conditions such as catatonia, bipolar mania, and schizophrenia. Interestingly, ECT also is likely to have efficacy in treating motor symptoms of $\mathrm{PD}^{41}$ and may be useful for PDP. Data from a small population of patients with medicationrefractory PDP who were treated with ECT showed a positive benefit in both motor and nonmotor symptoms as measured by the Brief Psychiatric Rating Scale and Hoehn and Yahr staging. ${ }^{42}$ Another small study of ECT in patients failing quetiapine treatment showed that bilateral ECT improved both motor features of PD as well as psychosis. The mechanism of this improvement is possibly through increases in regional blood flow as measured by SPECT in the right middle frontal gyrus after ECT. ${ }^{43}$ 
Alterations in nondopaminergic pathways are increasingly being recognized, and possibly contribute to motor and nonmotor symptoms of PD. ${ }^{28}$ Serotonergic neurons, largely originating in the dorsal raphe nuclei, are lost in PD and can demonstrate Lewy body formation. Indeed, there is some evidence that serotonin neurons are lost prior to degeneration of the midbrain dopaminergic pathways. ${ }^{29}$ Antipsychotics typically block dopamine receptors, and can cause possible extrapyramidal side effects, or worsening of motor function in PD. Newer a typical antipsychotics also have activity at serotonin receptors to varying degrees; however, in general, they also block dopaminergic receptors with risk of worsening motor function. There is evidence that $5-\mathrm{HT}_{2 \mathrm{~A}}$ receptors are increased in visual processing areas in the context of visual hallucinations and that binding is increased in the neocortex.$^{44}$ Hallucinogenic drugs such as phenyclidine and LSD (lysergic acid diethylamide) activate the $5-\mathrm{HT}_{2 \mathrm{~A}}$ receptor. This has prompted researchers to explore the use of selective $5-\mathrm{HT}_{2 \mathrm{~A}}$ receptor antagonists to potentially treat PDP without causing worsening motor function..$^{45}$ One such agent is pimavanserin, an inverse agonist without significant dopaminergic, muscarinic, noradrenergic, or histaminergic activity. A recent randomized placebo-controlled Phase III trial of pimavanserin demonstrated safety and general tolerability. Indices of psychosis were reduced with pimavanserin treatment by an effect size of $0.5 .{ }^{46}$ This is a promising new treatment.

Patients who have primary psychiatric disease with psychotic features and who also have comorbid PD or severe parkinsonism pose a challenge. Typically, antipsychotic doses for schizophrenia are significantly higher than those required for PDP; consequently, therapeutic antipsychotic doses may further exacerbate the motor symptoms. Additional systematic data remains necessary to provide treatment guidance in this unique population. A case report of a patient with schizophrenia who developed signs and symptoms of PD, as confirmed by Tc-99m-tropan-dopamine transporter SPECT, demonstrated this challenge. ${ }^{47}$ This patient was tried on individual trials of clozapine, quetiapine, and aripiprazole, which either did not control psychotic symptoms or worsened motor symptoms. Eventually, combination treatment with quetiapine and clozapine was found to be effective. This case exemplifies the complicated issues involved in treating primary psychosis in the context of PD.

\section{DSM-5 nomenclature}

In May 2013, an updated DSM-5 was released.$^{48}$ This updated document is used to diagnose psychiatric disease based on the most recent data regarding incidence, treatment, and epidemiology. Although there had been attempts to clarify diagnosis of PDP in the DSM-IV text revision era, PDP phenomena could be coded by mental health professionals depending on their view of the etiology. Diagnoses could include "Psychotic disorder due to a general medical condition," if deemed to be due to underlying PD, "Substanceinduced psychotic disorder" if second to dopamine agonist therapy, or "Psychosis NOS [not otherwise specified]" if felt to be due to possibly both or if the two were not able to be distinguished. Similar to DSM-IV, DSM-5 also includes "Psychosis due to another medical disorder" or "medicationinduced psychotic disorder," and states that the clinician using the diagnosis should have evidence that the psychotic phenomena are a "direct pathophysiological consequence of another medical condition." ${ }^{48}$ DSM-5 notes that "if the clinician has ascertained that the disturbance is due to both a medical condition and substance use, both diagnoses (ie, psychotic disorder due to another medical condition and substance/medication-induced psychotic disorder) can be given." ${ }^{48}$ As the etiology of PDP is not well understood, and may be multifactorial, with little direct evidence of causation, a more broad definition is likely to be, at this point in time, more accurate.

In contrast to DSM-IV, DSM-5 offers a newly delineated "neurocognitive disorder due to Parkinson's disease" to describe dementia secondary to $\mathrm{PD} .{ }^{48} \mathrm{~A}$ variety of nonmotor symptoms can be part of this diagnosis including apathy, depression, anxiety, personality, and psychotic symptoms; such features are supportive of this diagnosis, but are not a primary part of the diagnostic criteria. As evidence shows some association of cognitive changes with psychotic symptoms, this is an interesting diagnostic choice that would apply to many with PD who also have dementia, and is a more unified attempt at describing PDP. The multitude of possible diagnoses to describe this phenomenon highlights the state of our knowledge of PD. At present, the understanding of the etiology and pathogenesis of psychosis in the context of PD is not sophisticated enough to propose causation, and attempts to diagnose accurately reveal this challenge.

\section{Conclusion}

Nonmotor symptoms, especially psychotic symptoms, are commonly experienced by patients with PD, especially with advancing age, worsening cognition, and duration of illness. PDP can have serious consequences for quality of life for the individual with the disease and can contribute to caregiver burden. Development of a consensus definition 
of PDP will facilitate both clinical diagnosis and further research in understanding PDP. Clinicians are advised to routinely screen for psychotic symptoms in the office given that not all patients will report them. Medications may need to be modified accordingly. If reduction or elimination of medications cannot produce sufficient symptomatic benefit, or is associated with unacceptable decline in mobility, clinicians may consider the addition of an atypical antipsychotic such as quetiapine or clozapine to treat psychotic symptoms. Antipsychotic medications do have the risk of worsening motor symptoms, in addition to having metabolic and other adverse effects, and require monitoring. Treatment of psychotic symptoms could potentially allow patients to be managed in a home environment and improve quality of life.

\section{Disclosure}

Dr Stacy reports grant/research support from Michael J. Fox Foundation, National Institutes of Health, and Parkinson Study Group; he has been consultant to Acorda, Allergen, Chelsea, General Electric, Genzyme, Eli Lilly, Merz, Osmotica, Pfizer, ProStraken, SK Life Sciences, UCB, and Vanda; he was a member of CD-PROBE (Allergan) Protocol Steering Committee; has gained royalties from Informa Press for Handbook of Dystonia; and has been employed by Duke University School of Medicine. Dr Jakel reports no conflicts of interest in this work.

\section{References}

1. Goetz CG, Stebbins GT. Risk factors for nursing home placement in advanced Parkinson's disease. Neurology. 1993;43(11): 2227-2229.

2. Forsaa EB, Larsen JP, Wentzel-Larsen T, Alves G. What predicts mortality in Parkinson disease?: A prospective population-based long-term study. Neurology. 2010;75(14):1270-1276.

3. Archibald NK, Clarke MP, Mosimann UP, Burn DJ. Visual symptoms in Parkinson's disease and Parkinson's disease dementia. Mov Disord. 2011;26(13):2387-2395.

4. Factor SA, Feustel PJ, Friedman JH, et al. Longitudinal outcome of Parkinson's disease patients with psychosis. Neurology. 2003;60: 1756-1761.

5. Goetz CG, Fan W, Leurgans S, Bernard B Stebbins GT. The malignant course of "benign hallucinations" in Parkinson disease. Arch Neurol. 2006;63(5):713-716.

6. Rana AQ, Siddiqui I, Zangeneh M, Fattah A, Awan N, Yousuf MS. Predicting treatment-seeking for visual hallucinations among Parkinson's disease patients. Psychiatry Clin Neurosci. 2013;67(7):509-516.

7. Georgiev D, Danieli A, Ocepek L, et al. Othello syndrome in patients with Parkinson's disease. Psychiatr Danub. 2010;22(1):94-98.

8. Graff-Radford J, Ahlskog JE, Bower JH. Dopamine agonists and Othello's syndrome. Parkinsonism Relat Disord. 2010;16(10): 680-682.

9. Flann S, Shotbolt J, Kessel B, et al. Three cases of delusional parasitosis caused by dopamine agonists. Clin Exp Dermatol. 2010;35(7): $740-742$.
10. Kiziltan G, Ozekmekçi S, Ertan S, Ertan T, Erginöz E. Relationship between age and subtypes of psychotic symptoms in Parkinson's disease. J Neurol. 2007;254(4):448-452.

11. Fenelon G, Soulas T, Zenasni F, Cleret de Langavant L. The changing face of Parkinson's disease-associated psychosis: a Cross-sectional study based on the New NINDS-NIMH criteria. Mov Disord. 2010;25(6):763-766.

12. Fenelon G, Alves G. Epidemiology of psychosis in Parkinson's disease. J Neurol Sci. 2010;289(1-2):12-17.

13. Riedel O, Klotsche J, Spottke A, et al. Frequency of dementia, depression, and other neuropsychiatric symptoms in 1,449 outpatients with Parkinson's disease. J Neurol. 2010;257(7):1073-1082.

14. Rabey JM. Hallucinations and psychosis in Parkinson's disease. Parkinsonism Relat Disord. 2009;15 suppl 4:S105-S110.

15. Ravina B, Marder K, Fernandez HH, et al. Diagnostic criteria for psychosis in Parkinson's disease: report of an NIND, NIMH work group. Mov Disord. 2007;22:1061-1068.

16. Morgante L, Colosimo C, Antonini A, et al. Psychosis associated to Parkinson's disease in the early stages: relevance of cognitive decline and depression. J Neurol Neurosurg Psychiatry. 2012;83:76-82.

17. Factor SA, Steenland NK, Higgins DS, et al. Disease-related and genetic correlates of psychotic symptoms in Parkinson's disease. Mov Disord. 2011;26(12):2190-2195.

18. Zhou MZ, Gan J, Wei YR, Ren XY, Chen W, Liu ZG. The association between non-motor symptoms in Parkinson's disease and age at onset. Clin Neurol Neurosurg. 2013;115(10):2103-2107.

19. Yoritaka A, Shimo Y, Takanashi M, et al. Motor and non-motor symptoms of 1453 patients with Parkinson's disease: Prevalence and risks. Parkinsonism Relat Disord. 2013;19:725-731.

20. Leroi I, Pantula H, McDonald K, Harbishettar V. Neuropsychiatric symptoms in Parkinson's disease with mild cognitive impairment and dementia. Parkinsons Dis. 2012;2012:308097.

21. Mack J, Rabins P, Anderson K, et al. Prevalence of psychotic symptoms in a community-based Parkinson disease sample. Am J Geriatr Psychiatry. 2012;20(2):123-132.

22. Lee AH, Weintraub D. Psychosis in Parkinson's disease without dementia: common and comorbid with other non-motor symptoms. Mov disord. 2012;27(7):858-863.

23. Munholz RP, Espay AJ, Morgante F, et al. Long-duration Parkinson's disease: role of lateralization of motor features. Parkinsonism Relat Disord. 2013;19(1):77-80.

24. Sawada H, Oeda T, Yamamoto K, et al. Trigger medications and patientrelated risk factors for Parkinson disease psychosis requiring anti-psychotic drugs: a retrospective cohort study. BMC Neurol. 2013;13:145.

25. Ecker D, Unrath A, Kassubek J, Sabolek M. Dopamine agonists and their risk to induce psychotic episodes in Parkinson's disease: a casecontrol study. BMC Neurol. 2009;9(1):23.

26. Fenelon G, Goetz CG, Karenberg A. Hallucinations in Parkinson disease in the prelevodopa era. Neurol. 2006;66:93-98.

27. Ravina B, Marek K, Eberly S, et al. Dopamine transporter imaging is associated with long-term outcomes in Parkinson's disease. Mov disord. 2012;27(11):1392-1397.

28. Huot P, Fox SH. The serotonergic system in motor and non-motor manifestations of Parkinson's disease. Exp Brain Res. 2013;230: 463-476.

29. Braak H, Ghebremedhin E, Rüb U, Bratzke H, Del Tredici K. Stages in the development of Parkinson's disease-related pathology. Cell Tissue Res. 2004;318:121-134.

30. Marras C, Kopp A, Qiu F, et al. Antipsychotic use in older adults with Parkinson's disease. Mov Disord. 2007;22:319-323.

31. Widge AS, Agarwal P, Giroux M, Farris S, Kimmel RJ, Hebb AO. Psychosis from subthalamic nucleus deep brain stimulator lesion effect. Surg Neurol Int. 2013;4:7.

32. Smith DJ, Langan J, McLean G, Guthrie B, Mercer SW. Schizophrenia is associated with excess multiple physical-health comorbidities but low levels of recorded cardiovascular disease in primary care: cross-sectional study. BMJ Open. 2013;3(4). 
33. Llebaria G, Pagonabarraga J, Martinez-Corral M, et al. Neuropsychological correlates of mild to severe hallucinations in Parkinson's disease. Mov Disord. 2010;25(16):2785-2791.

34. Lewis SJG, Shine JM, Duffy S, Halliday G, Naismith SL. Anterior cingulate integrity: executive and neuropsychiatric features in Parkinson's disease. Mov Disord. 2012;27(10):1262-1267.

35. Fernandez HH, Trieschmann ME, Okun MS. Rebound psychosis: effect of discontinuation of antipsychotics in Parkinson's disease. Mov Disord. 2005;20:104-115.

36. Seppi K, Weintraub D, Coelho M, et al. The movement disorder society evidence-based medicine review update: treatments for the nonmotor symptoms of Parkinson's disease. Mov Disord. 2011;26(S3): S42-S80.

37. Merims D, Balas M, Peretz C, Shabtai H, Giladi N. Rater-blinded, prospective comparison: quetiapine versus clozapine for Parkinson's disease psychosis. Clin Neuropharmacol. 2006;29(6):331-337.

38. Rabey JM, Prokhorov T, Miniovitz A, Dobronevsky E, Klein C. Effect of quetiapine in psychotic Parkinson's disease patients: a double-blind labeled study of 3 months' duration. Mov Disord. 2007;22:313-318.

39. Shotbolt P, Samuel M, Fox C, David AS. A randomized controlled trial of quetiapine for psychosis in Parkinson's disease. Neuropsychiatr Dis Treat. 2009;5:327-332.

40. Tagai K, Nagata T, Shinagawa S, Tsuno N, Ozone M, Nakayama K. Mirtazapine improved visual hallucinations in Parkinson's disease: a case report. Psychogeriatrics. 2013;13(2):103-107.
41. Andersen K, Balldin J, Gottfries CG, et al. A double-blind evaluation of electroconvulsive therapy in Parkinson's disease with "on-off" phenomena. Acta Neurol Scand. 1987;76(3):191-199.

42. Ueda S, Koyama K, Okubo Y. Marked improvement of psychotic symptoms after electroconvulsive therapy in Parkinson disease. JECT. 2010;26(2):111-115.

43. Usui C, Hatta K, Doi N, et al. Improvements in both psychosis and motor signs in Parkinson's disease, and changes in regional cerebral blood flow after electroconvulsive therapy. Prog NeuroPsychopharmacol Biol Psychiatry. 2011;35:1704-1708.

44. Ballanger B, Strafella AP, van Eimeren T, et al. Serotonin 2A receptors and visual hallucinations in Parkinson's disease. Arch Neurol. 2010;67: 416-421.

45. Friedman JH. Pimavanserin for the treatment of Parkinson's disease psychosis. Expert Opin Pharmacother. 2013;14(14):1969-1975.

46. Cummings J, Isaacson S, Mills R, et al. Pimavanserin for patients with Parkinson's disease psychosis: a randomized, placebo-controlled phase 3 trial. Lancet. Epub October 31, 2013.

47. Lan CC, Su TP, Chen YS, Bai YM. Treatment dilemma in comorbidity of schizophrenia and idiopathic Parkinson's disease. Gen Hosp Psychiatry. 2011;33(4):411.

48. Diagnostic and Statistical Manual of Mental Disorders, Fifth Edition [homepage on the Internet]. Arlington: American Psychiatric Association; 2013. Available from: dsm.psychiatryonline.org. Accessed June 1, 2013.
Journal of Parkinsonism \& Restless Legs Syndrome

\section{Publish your work in this journal}

Journal of Parkinsonism and Restless Legs Syndrome is an online, open access, peer-reviewed journal. The journal publishes review articles, historical reviews, original research articles, case reports, letters to the editor, clinical teaching cases, neuroradiology highlights, neuropathology highlights, neuropsychiatry highlights, autobiographies, conference

\section{Dovepress}

proceedings, abstracts and book reviews. The manuscript management system is completely online and includes a very quick and fair peerreview system, which is all easy to use. Visit http://www.dovepress.com/ testimonials.php to read real quotes from published authors. 